\title{
Force Sensing for Multi-legged Walking Robots: Theory and Experiments - Part 2: Force Control of Legged Vehicles
}

\author{
A. Schneider, U. Schmucker \\ Fraunhofer Institute for Factory Operation and Automation \\ Germany,
}

\section{Use of force information in legged vehicle control}

\subsection{Main approaches and principles of force control}

Approaches to manipulator control using force information can be subdivided into two major groups. The first group uses logic branching of the control when the measured force satisfies certain conditions. The second group introduces continuous force feedback as an explicit force control or active force feedback method.

The basic approaches to force feedback control that are already used or can be applied to walking robot motion control are discussed in many studies (Raibert \& Craig, 1981; Gorinevsky et al., 1997; Gurfinkel et al., 1982, 1984; Mason \& Salisbury, 1985; De Schutter, 1986, De Schutter \& Brussel, 1988) and papers. Whitney (Whitney, 1977, 1987) was a pioneer of force control.

Stiffness control (Raibert\& Craig, 1981). The simplest method of stiffness control is linear force feedback of the form:

$$
U=c\left(F-F_{d}\right),
$$

where $U$ is a voltage of drive, is an applied force, $F$ is a reference force and c is a com pliant force sensor. If the force sensor is stiff, this feedback is equivalent to high-gain position feedback. The damping naturally present in the system may be insufficient for such feedback, thus resulting in a highly oscillatory system. To increase the damp ing, a velocity feedback should be introduced in the system.

Active or artificial compliance. This method was developed in the late nineteen seventies and early eighties for use in robotic systems as well as for six-legged robots (Whitney,1977; McGhee et al., 1980; Klein \& Briggs, 1980; Devjanin et al., 1982; Salisbury \& Craig, 1982; Gurfinkel et al., 1984; De Schutter \& Brussel, 1988). The simplest law of this method is the form:

$$
x-x_{d}=c\left(F-F_{d}\right)
$$

where $x$ is a coordinate of end-effector, $x_{d}$ is a reference coordinate, $F$ is an applied force, $F_{d}$ is a reference force and $c$ is a desired compliance.

In (Klein \& Briggs, 1980), it is applied to six-legged OSU hexapod force control in the law form:

$$
\dot{z}=\dot{z}_{p}+g_{1}\left(z_{p}-z\right)+g_{F}\left(\dot{F}_{p}-\dot{F}\right),
$$


where $z$ and $\dot{z}$ are the actual vertical leg position and velocity, $z_{p}$ and $\dot{z}_{p}$ are the desired vertical position and velocity and $g_{1}, g_{\mathrm{F}}$ are gains, respectively.

In (Golubev et al., 1979; Devjanin et al., 1982; Gorinevsky \& Shneider, 1987), a some-what different law is used so that an external force acting on the legs of the robot "Masha" will cause a displacement of the end-effector. Fig. 1.1 shows the interaction of the positional and force elements of the control system for a leg of the walking robot "Katharina".

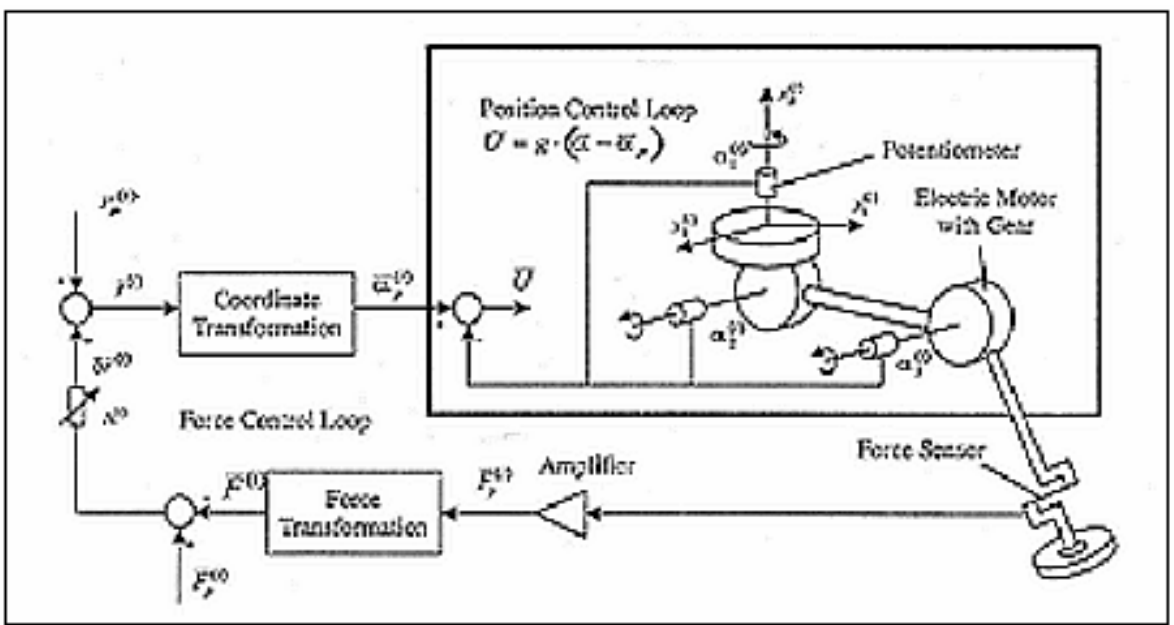

Fig. 1.1. Flow chart of leg force control.

In order to understand the behavior of a system with force feedback, assume that the servo systems track the commanded coordinates to the leg tips with high accuracy.

Write the radius vector $\bar{r}^{(i)}$ of the $i$-leg in the body's fixed coordinate system as $\bar{r}^{(i)}=\bar{r}_{p}^{(i)}+$ $\delta \bar{r}^{(i)}$, where $\bar{r}_{p}^{(i)}$ is the commanded position calculated by the leg motion control algorithms and $\bar{r}_{p}^{(i)}$ is the force correction. The force feedback transmits the leg position to the positional servo system. The leg position differs from $\delta \bar{r}^{(i)}$ as:

$$
\delta \bar{r}^{(i)}=\Lambda^{(i)}\left(\bar{N}^{(i)}-\bar{N}_{p}^{(i)}\right),
$$

where $\Lambda^{(i)}$ is the symmetric positive definite feedback gain matrix and $\bar{N}^{(i)}$ and $\bar{N}_{p}^{(i)}$ are actual and commanded force vectors, respectively. If the force acting on the leg differs from the commanded value, it causes additional leg displacement proportional to the difference. Such system behavior is similar to that of an elastic spring with a compliance of $\Lambda^{(i)}$ Active compliance can be controlled by varying the elements of the matrix $\Lambda^{(i)}$.

The active compliance method is widely used to control the motion of walking robots' legs and bodies (Klein \& Briggs, 1980; Devjanin et al., 1982; Gorinevsky \& Shneider, 1990; Alexandre et al., 1998). For instance, the active compliance of a leg for the walking robots "Masha" and "Katharina" was in a range of $0.01 \mathrm{~cm} / \mathrm{N}$ to $0.03 \mathrm{~cm} / \mathrm{N}$.

Active accommodation or generalized damping control (Whitney,1977; Mason \& Salisbury, 1985; Schmucker et al., 1997). The desired end-effector behavior is also often specified as damper behavior in the form 


$$
\dot{x}-v_{d}=g\left(F-F_{d}\right),
$$

where $\dot{x}$ is an end-effector velocity, $v_{d}$ is a reference velocity, $F_{d}$ is a reference force, and $g$ is a damping factor. It follows that an externally applied constant force acting on the endeffector will generate a steady state motion with a velocity proportional to the force.

The theoretical analysis of this method and experiments has been treated many times over including in (Gorinevsky et al., 1997).

The active accommodation method based on information on the main force and torque vectors acting on the vehicle body has been used for plane-parallel displacements and orientation changes of the body with resting support feet. Let the commanded vectors of linear and angular body velocities depend uniquely (for example, linearly) on the external force and torque as in

$$
\bar{V}=G_{f}\left(\bar{F}-\bar{F}_{p}\right), \bar{\omega}=G_{\varpi}\left(\bar{M}-\bar{M}_{p}\right),
$$

where $\bar{V}, \bar{\omega}$ are the measured and commanded values of linear and angular body velocities, respectively, and $G_{f}, G_{\omega}$ are the matrices of accommodation. If a body's commanded vector velocities $(\bar{V}, \bar{\omega})$ depend on the external force or torque, then a vehicle body will be displaced or turned corresponding to the "accommodation".

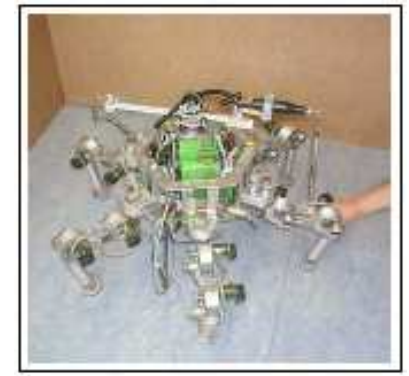

(a) Downward-motion of body

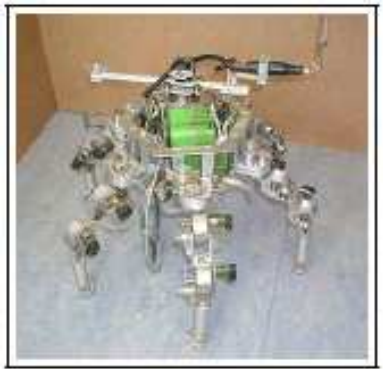

(b) Upward-motion of body

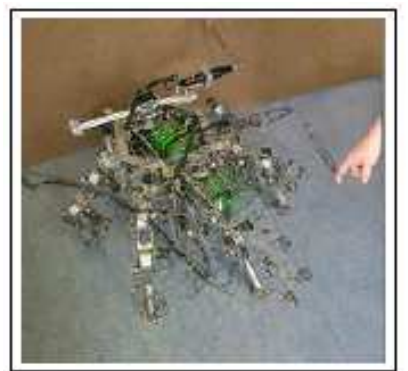

(c) Left-right motion of body

Fig. 1.2. "Katharina”.

Figures 1.2a - 1.2c show the plane-parallel displacements of the body of the robot "Katharina" due to an external force. The dynamometer imposed the force parallel to the Cartesian coordinates related to the body. The displacement of the body was a response to the external reaction.

Impedance control. The relation between the external force and manipulator motion can generally be specified as a desired impedance of the manipulator (Hogan, 1985; Kazerooni et al., 1986; De Schutter \& Brussel, 1988; Tzafestas et al., 1995; Palis et al., 2001).

The impedance can be defined as a transfer function between the external force acting on the manipulator and its displacement. The specified impedance can be achieved by different means using implicit or explicit force control.

Hybrid position/force control (Raibert\& Craig, 1981; Salisbury \& Craig, 1982; Sinha \& Goldenberg, 1993). Some degrees of freedom of the end-effector are position controlled and others are force controlled. This method is based on the concept of a selection matrix, which is a diagonal 6x6 matrix with zeros and ones on the diagonal. Artificial Neural Networks $(A N N)$ These networks include a large variety of control methods (Haykin, 1994). The 
"Cerebeller modeled articulation controller" is one example of an ANN that has been used for the hybrid position/force control of a quadruped (Frik, 1996; Lin \& Song, 1997; Cruse et al., 1998).

Force control in theory and practice involves a number of different force control methods, surveys of which can be found, for example, e.g., in (De Schutter, 1986; Gorinevsky et al., 1997; Sciavicco \& Siciliano, 2000; Surdilovic \& Vukobratovic, 2001).

\subsection{Force control for step adaptation}

Moving a vehicle over structured terrain requires adapting each leg to different ground clearance. The step cycle must be modified in order to obtain the correct ground contact. Foot force information is used to obtain the ground contact information.

While there is contact with the ground, the foot force increases as a function of the ground properties: quickly for solid ground, slowly for soft ground. The ground contact phase ends when the desired foot force distribution is attained. Together with active compliance, this produces an adaptable step. Analyzing the foot force dependent on foot position enables measuring information about soil softness. This is needed to adjust the step cycle in the transfer phase for sufficient foot clearance.

A similar algorithm is used for obstacle detection and navigation. During the transfer phase, the touch detection algorithm is activated in the direction of transfer. An obstacle is detected if the foot force reaches a predefined value. At this moment, the foot should be stopped (Devjanin et al., 1983).

When obstacle detection is combined with active compliance, the foot begins to stop as the acting force increases and before the force level for obstacle detection is reached. In this case, a hard impact against an obstacle is prevented.

\subsection{Use of the active compliance method for force component distribution of legs}

The coordinate system $O X_{1} X_{2} X_{3}$ (Fig. 1.3)is used to describe leg motion.

Assume the voltages of the leg drives are

$$
\bar{U}^{(i)}=G_{1}^{(i)}\left(\bar{V}_{p}^{(i)}-\bar{V}^{(i)}\right),
$$

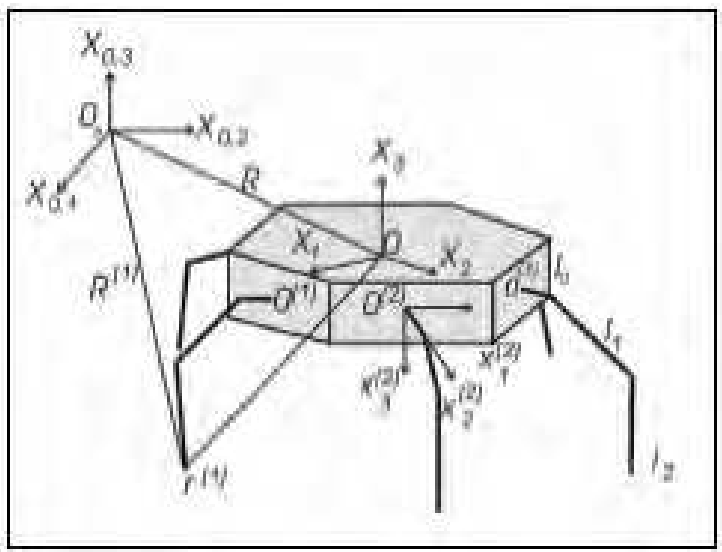

Fig. 1.3. Diagram of vehicle and coordinate systems. 
where the program velocity vector is:

$$
\bar{V}_{p}^{(i)}=-G_{2}^{(i)}\left(\bar{r}^{(i)}-\bar{r}_{p}^{(i)}\right)-G_{3}^{(i)}\left(\bar{N}^{(i)}-\bar{N}_{p}^{(i)}\right)
$$

Here, $i$ is the leg number and $G_{1}^{(i)}, G_{2}^{(i)}, G_{3}^{(i)}$ are the (3x3) feedback gain matrices of velocity, position and force reaction, respectively. $\bar{V}_{p}^{(i)}, \bar{V}^{(i)}$ are commanded and measured velocity vectors, $\bar{r}^{(i)}, \bar{r}_{p}^{(i)}$ i.e. radius vectors of realized and commanded position of $i$-th leg (vector $\bar{r}^{(i)}$ is determined by the joint angles), and $\bar{N}^{(i)}-\bar{N}_{p}^{(i)}$ are measured and commanded values of the force reaction in $i$-th leg. Expression (1.7) can be transformed into

$$
\bar{U}^{(i)}=-G_{r}^{(i)}\left[\left(\bar{r}^{(i)}-\left(\bar{r}_{p}^{(i)}+\delta \bar{r}^{(i)}\right)\right]-G_{1}^{(i)} \bar{V}^{(i)}\right.
$$

where $\delta \bar{r}^{(i)}$ is equal (1.4), and $G_{r}^{(i)}=G_{1}^{(i)} G_{2}^{(i)}$. Vector $\delta \bar{r}^{(i)}$ can be considered a position correction of the commanded position of $i$-th leg tip, $\Lambda^{(i)}=\left(G_{2}^{(i)}\right)^{-1} G_{3}^{(i)}$ is the matrix of the mechanical compliance of the leg. Value $\bar{r}_{*}^{(i)}=\bar{r}_{p}^{(i)}+\delta \bar{r}^{(i)}$ denotes a commanded position input to; the servo system.

It follows from (1.5) and (1.8), that when a measured force reaction $\bar{N}^{(i)}$ coincides with a commanded force reaction $\bar{N}_{p}^{(i)}$, the leg tip position $\bar{r}_{*}^{(i)}$ is equal to the commanded value $\bar{r}_{p}^{(i)}$. Since the leg joints of the experimental walking vehicles ("Masha" and "Katharina") are equipped with joint angle potentiometers, the commanded trajectories $\bar{r}_{*}^{(i)}$ notated as Cartesian coordinates have to be transformed into commanded joint angles $\bar{\alpha}^{(i)}$.

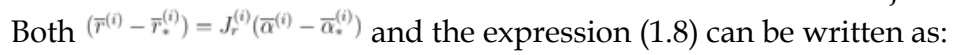

$$
\bar{U}^{(i)}=G_{r}^{(i)} J_{r}^{(i)}\left(\bar{\alpha}^{(i)}-\left(\bar{\alpha}_{*}^{(i)}\right)-G^{(i)} \dot{\bar{\alpha}}^{(i)},\right.
$$

Where $J_{r}^{(i)}$ is the Jacobean from the joint angles to the Cartesian coordinates of the leg tip and $\bar{\alpha}^{(i)}$ is the three-component vector of measured joint angle values. In this case, the velocity feedback is not effective because the drives have high damping due to their high reduction. Consequently, in (1.9), $G^{(i)}=0$ and the expression (1.9) for voltage can be written as

$$
\bar{U}^{(i)}=G_{2}^{(i)}\left(\bar{\alpha}^{(i)}-\bar{\alpha}_{*}^{(i)}\right),
$$

where $G_{2}^{(i)}=G_{r}^{(i)} J_{r}^{(i)}$ is the feedback gain matrix of the position.

The coordinate system $O X_{1} X_{2} X_{3}$, rigidly connected with the body of the robot and a world coordinate system $O X_{01} X_{02} X_{03}$ are used to evaluate algorithms for the control of foot reaction forces. The desired motion of the body can be described by a radius vector $\bar{R}_{p}$, describing the desired position of the body's center (point0), and a matrix $A_{p}^{(i)}$, consisting of the directional cosines between $O X_{1} X_{2} X_{3}$ and $O X_{01} X_{02} X_{03}$.

Assuming no slippage occurs between ground and feet, the programmed positions of the ends of supporting legs have to be specified according to

$$
\bar{R}_{p}+A_{p}^{(i)} \cdot \bar{r}_{p}^{(i)}=\bar{R}^{(i)}
$$

where $\bar{R}^{(i)}$ is the radius vector of $i$-th foot contact point in the system $O X_{01} X_{02} X_{03}$.

Let the movement of the robot be controlled according to the formulas (1.5), (1.8) and (1.9), i.e. the feet have an artificial compliance. The robot's actual movement will then be 
somewhat different than programmed. This means the radius vector $\bar{r}^{(i)}$ of the end points of the feet differs from $\bar{r}_{p}^{(i)}$ and (1.11) can be replaced by (1.8):

$$
\bar{R}+A^{(i)} \cdot \bar{r}^{(i)}=\bar{R}^{(i)}
$$

where $\bar{R}$ is the radius vector of the actual position of central point $0 ; A^{(i)}$ is the actual matrix of directional cosines between $O X_{1} X_{2} X_{3}$ and $O X_{01} X_{02} X_{03}$.

If the deviation between programmed and actual paths is small, then, according to (1.11) and (1.12), the following equation holds for the supporting legs with an accuracy up to second order terms:

$$
\Delta \bar{R}+\Delta \bar{\chi} \times \bar{r}_{p}^{(i)}+\Delta \bar{r}^{(i)}+\Delta \bar{r}_{0}^{(i)}+\delta \bar{r}^{(i)}=0
$$

Here, $\Delta \bar{R}=A^{-1}\left(\bar{R}-\bar{R}_{p}\right)$ is the radius vector, characterizing a small linear deviation of point 0 from the programmed value, $\Delta \bar{\chi}$ is the vector of small angular deviation of body orientation, $\Delta \bar{r}^{(i)}$ is the radius vector of foot deflection due to elastic deformations, $\Delta \bar{r}_{0}^{(i)}$ is the radius vector of the error affected by the control system and $\delta \bar{r}^{(i)}$ is the correction value for the foot deflection added to the programmed value calculated from the force feedback according to (1.5).

Vectors $\Delta \bar{r}^{(i)}, \Delta \bar{r}_{0}^{(i)}, \delta \bar{r}^{(i)}$ are given in the coordinate system $O X_{1} X_{2} X_{3}$. Supporting forces $\bar{N}^{(i)}$ in the $i$-th leg and their elastic deformations are connected by $\Delta \bar{r}^{(i)}=\left(C^{(i)}\right)^{(i)} \bar{N}^{(i)}$, where $C^{(i)}$ is a positive definite matrix of the leg's mechanical stiffness.

The following assumes that the foot deflection $\delta \bar{r}^{(i)}$ resulting from artificial compliance is much larger than the deflections $\Delta \bar{r}^{(i)}, \Delta \bar{r}_{0}^{(i)}$. Neglecting their influence, the force distribution equation (1.13) can be written as:

$$
\Delta \bar{R}+\Delta \bar{\chi} \times \bar{r}_{p}^{(i)}+\delta \bar{r}^{(i)}=0
$$

Assuming the walking robot moves slowly, the influence of dynamic factors on the force distribution may be neglected too. The static equilibrium conditions are added to equation (1.5):

$$
\sum_{i=1}^{6}\left(\bar{r}_{p}^{(i)}+\delta \bar{r}^{(i)} \times \bar{N}^{(i)}=\bar{M}, \sum_{i=1}^{6} \bar{N}^{(i)}=\bar{N}\right.
$$

where $\bar{N}$ is gravitational force and $\bar{M}$ is the general torque resulting from the gravitational force in a body's fixed coordinate frame.

Conditions (1.5), (1.14), (1.15) yield a closed system of equations to determine $\Delta \bar{R}$, $\Delta \bar{\chi}, \delta \bar{r}^{(i)}, \bar{N}^{(i)}$. The body's deviation from the nominal position (vectors $\Delta \bar{R}, \Delta \bar{\chi}$ ), the reactions $\bar{N}^{(i)}$ in the supporting legs and the vectors $\delta \bar{r}^{(i)}$ are defined by the parameters $\left.\bar{r}_{p}^{(i)}, \bar{N}_{p}^{(i)}, \Lambda^{(i)}\right)$. The values $\bar{r}_{p}^{(i)}$, which define the programmed movement of the leg ends, are calculated based on the position control element in the control system. The additional values $\delta \bar{r}^{(i)}$ according to (1.5) are calculated in the force control element of the entire system. Force feedback should occur with constant coefficients $\Lambda^{(i)}$. If the programmed movements of the legs (vectors $\bar{r}_{p}^{(i)}$ ) and the compliance matrix $\Lambda^{(i)}$ are given, the 
programmed reaction forces $\bar{N}_{p}^{(i)}$, which can be provided as an input to the system, uniquely define the support forces $\bar{N}^{(i)}$ and the deviation of the body from the nominal position. According to (1.5), (1.14) and (1.15), the robot's actual movement coincides with the programmed movement $(\Delta \bar{R}=0, \Delta \bar{\chi}=0)$ if the programmed reaction forces $\bar{N}_{p}^{(i)}$ meet the static equilibrium conditions. In this case, the actual reaction forces $\bar{N}^{(i)}$ are identical to the programmed values $\bar{N}_{p}^{(i) !}$.

\subsection{Distribution of vertical force components}

First, we shall consider a situation in which the robot moves forward with a three point gait with a step length of about $10 \mathrm{~cm}$ over an even solid surface. There is no force control of distribution. The vertical force components of support reactions in the legs are plotted in Fig.1.4. Since the system is statically indeterminate with respect to the forces acting on the legs (more than three legs can be on a support) and the control system lacks force feedback, support reactions change randomly. This can lead to significant mechanical loading on separate legs.

The force distribution control essentially improves the robot's pattern of locomotion and increases its stability of motion.

Force distribution can be determined by several methods within the framework of static indeterminacy. The situations in which the walking robot moves relatively slowly have been considered and therefore the influence of dynamic factors on force distribution may be disregarded. Assuming the support surface is slightly uneven and the vehicle body is in the horizontal position, then the commanded horizontal force components are zero. The commanded vertical force components were computed from the body's orientation relative to the gravity vector and from the leg configuration.

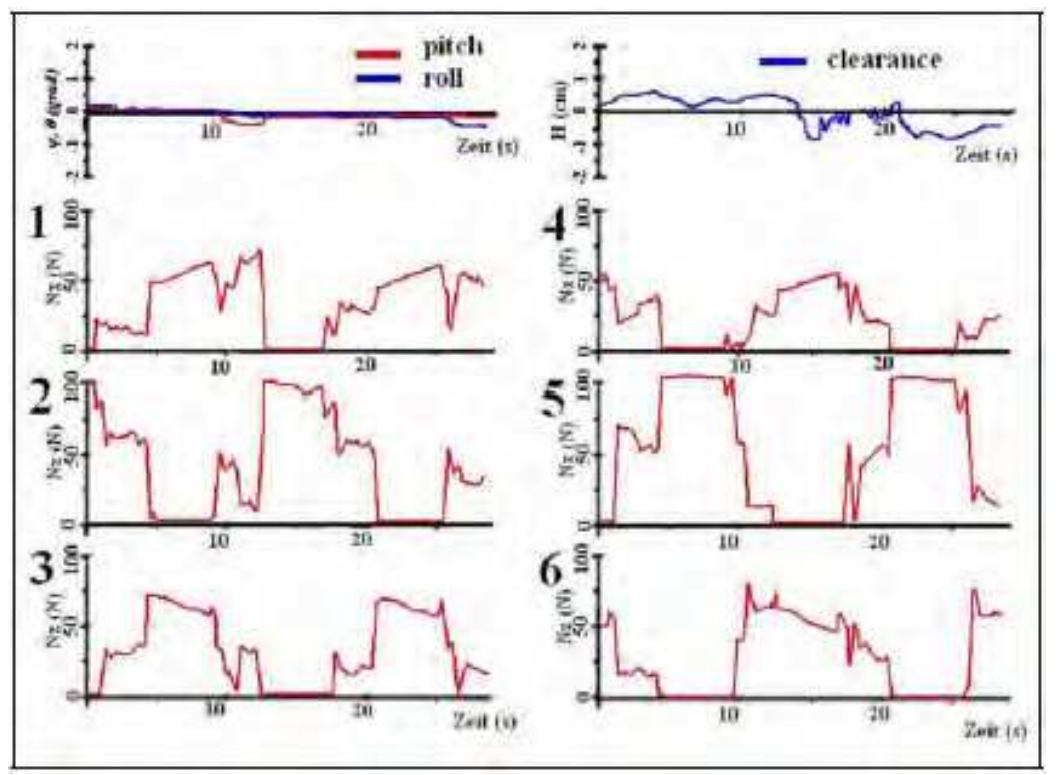

Fig. 1.4. Experimental results of foot forces in locomotion over solid surface. 
Let the horizontal force components be zero. Then the vertical force components must satisfy the static equilibrium equations:

$$
\sum_{i \in I} N_{z}^{(i)}=P, \sum_{i \in I} N_{z}^{(i)} x^{(i)}=P X, \sum_{i \in I} N_{z}^{(i)} y^{(i)}=P Y,
$$

where $P$ is the vehicle weight, $x^{(i)}, y^{(i)}$ are coordinates of the $i$-th leg tip and $X, Y$ are coordinates of the vehicle center of mass. Summation in equations (1.16) is performed over set of $I$ the supporting legs.

Forces in $n$ supporting legs should satisfy the three equations in (1.16). If $n>3$, then there is more than one unique solution. There are different ways to eliminate the indeterminacy. Assume the vertical force components are required to satisfy

$$
\sum_{i \in I}\left(N_{z}^{(i)}\right)^{2} \rightarrow \min
$$

The purpose of this condition is energy optimization (Klein \& Wahavisan, 1984). The exact condition of energy optimization is more complex and has been considered in (McGhee et al., 1980; Okhotsimsky \& Golubev, 1984).

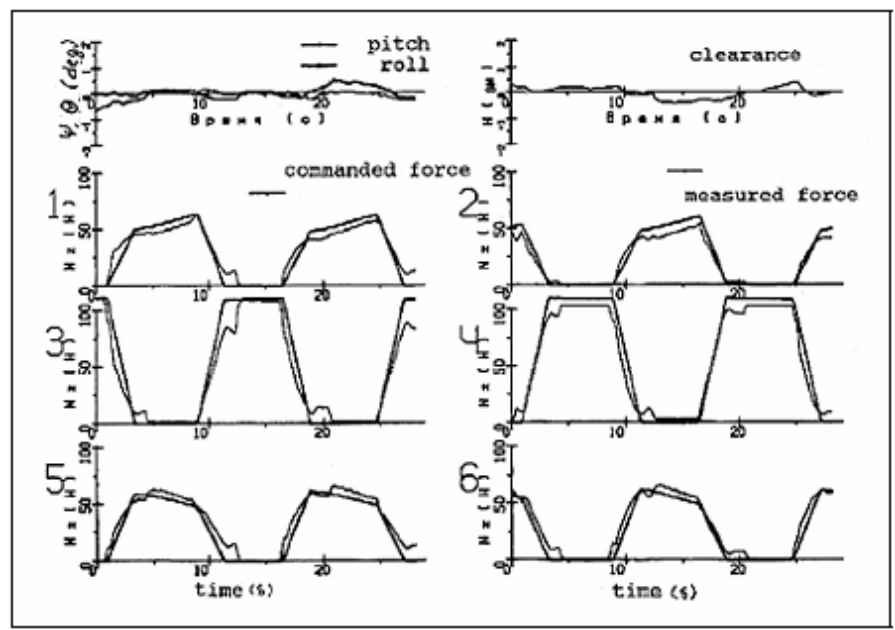

Fig. 1.5. Experimental results of vertical forces distribution.

Solving the equations (1.16) requires calculating the coordinates $(X, Y)$ of the vehicle center of mass in terms of leg configuration. The simplified model of mass distribution contains the legs with the mass point $\mathrm{m}$ located on the leg ends and the body's mass $M_{B}=M-6 \mathrm{~m}$, where $M$ is the total mass of the vehicle (Fig.1.6)

(Gorinevsky \& Shneider,1990). The solution of equation (1.16) under condition (1.17) can be obtained by LaGrange multiplier method (Gantmacher, 1960).

Experimental results have been obtained for the locomotion of the multi-legged robot "Masha" over an even, solid surface with tripod gait motion. The experimental results of the control of vertical force distribution in robot locomotion are plotted in Fig. 1.5. During the joint support phase, one set of legs is loaded and another is unloaded. Loads are redistributed smoothly, without jumps, as opposed to a situation in which forces are not controlled. More detailed results are presented in (Gorinevsky \& Shneider, 1990). 


\subsection{Constrained motion control}

The robot's body and the work tools attached to it can be used to perform manipulation tasks. In such cases, it is necessary to control the force reactions and position or velocity of tool motions along constraints. Required motions can be achieved, for instance, by adjusting the matrix elements of a force feedback control law depending on programmed or actual movements. Many service and process operations may be considered motions with a mechanical constraint imposed on the manipulated objects. Control algorithms for manipulator systems with motion along constraints are discussed in numerous studies (Mason \& Salisbury, 1985; De Schutter \& Brussel, 1988; Gorinevsky et al., 1997; Lensky et al., 1986).

Similar situations arise out of the motion control of legged platforms as manipulation robots. Such operations should be performed by control of contact forces. Hence, it is necessary to have a force system which measures the vectors of main force $(\bar{F})$ and torque $(\bar{M})$ ) acting on the robot (see section 7.1).

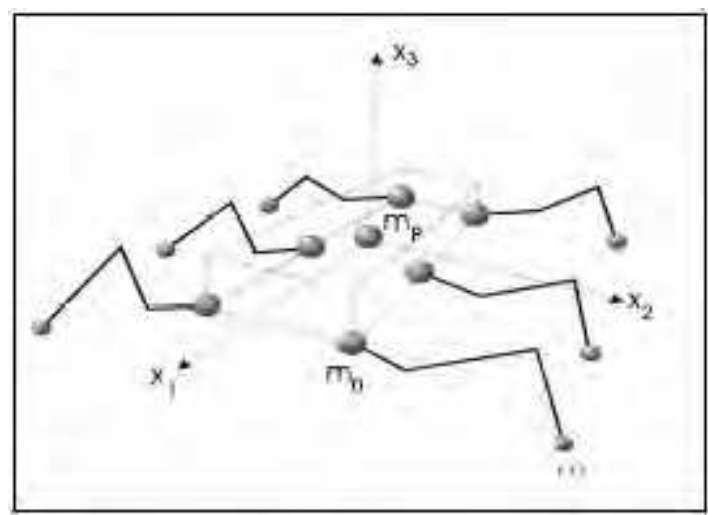

Fig. 1.6. Simplified model mass.

The generalized approach to synthesizing manipulator system motion along an object contour as a superposition of two basic motions at the normal and tangent to the contour is described (Gorinevsky et al., 1997). A force sensor controls end-effector motion in the directions normal to the constrain.

A motion "along the constraint", i.e. tangential to the constraint, is under positional control. The basic motion motion is maintaining contact with an object. Accordingly, the "desired" velocity vector $\bar{V}_{p}$ of a tool or manufacturing equipment is as a sum of two terms:

$$
\bar{V}_{p}=\bar{V}_{n}+\bar{V}_{\tau}=g\left(F_{n}-F_{p}\right) \bar{n}+V \overline{\text { tau }}
$$

The first term $\bar{V}_{n}$ in (1.18) is a vector directed towards the object if $F_{n}<F_{p}$ and from the object if $F_{n}>F_{p}$. The second component $\bar{V}_{\tau}=$ const $>0$ in the expression (1.18) is the programmed velocity tangential to the surface of the object (e.g. stick, tool). $F_{p}=$ const $>0$ is the programmed value of the normal component of the force with which, for example, a tool presses against the surface of an object, $F_{n}$ is the normal force vector, component $\bar{n}$ and $\overline{t a u}$ are the normal and tangential unit vectors, $\lambda>0$ is a constant feedback gain and is a commanded value of the tool velocity tangential to the surface of the object.

If the friction between the tool and the surface is absent or there is Coulomb friction, the vectors $\bar{n}$ and $\overline{t a u}$ can be determined from a force sensor. 
For many service operations such as assembly, moving tools along a surface, opening hatches, rotating various handles, etc., it is expedient to use the law of (1.18). In other cases when there is no movement along the constraint, e.g. drilling operations, it is possible to assume $v \bar{\tau}=0$.

If the constraint is not known exactly, the motion of the body with the manipulator can be extended in all directions. In this case, the body's compliance motion can be implemented with active compliance or damping controls. Relationship (1.18) represents a nonlinear method of control. Although $\bar{V}_{n}$ is calculated using linear force feedback, the control law (1.18) also involves the product $\bar{V} \cdot \bar{n}$.

These force control approaches have been used in problems of locomotion, to maneuver walking robots and for various service operations (Schmucker et al., 1997; Schneider \& Schmucker, 2000).

\section{Locomotion over soft soil and soil consolidation}

A ground deformation under the supporting leg leads to a modification of robot motion. Vertical sinking of supporting feet into a soft soil necessitates maintaining the correct position of the body (angular and vertical velocities) relative to the bearing surface during every step. A shear deformation of the ground leads to reduced vehicle velocity in absolute space (Bekker, 1969; Okhotimsky et al.,1985; Wong, 1993; Gaurin, 1994).Thus, it is necessary to work out the method and algorithms for locomotion over soft soil and stabilization of the vehicle body.

\subsection{Determination of mechanical properties of soil by means of legs}

In an investigation of ground passability, the resistance to compressive and shear forces can be used to measure ground bearing capabilities. One of the most widely used ground bearing capability measurement devices is the bevameter (Bekker, 1969; Wong, 1993; Kemurdjian \& et. al., 1993; Manko, 1992).

A bevameter consists of a penetration plate and drive to implement various dependencies between the penetration plate's sinking and lateral shift and also to record the compressive load and shear. To obtain a more accurate picture of ground bearing while a vehicle is moving, the dimensions and form of the penetration plate should correspond to its support surface. The walking vehicle leg equipped with a foot force sensor and a joint angle potentiometer constitutes an ideal bevameter. The laboratory robot "Masha" was used to study soil properties (Gorinevsky \& Shneider, 1990; Schneider \& Schmucker, 2001).

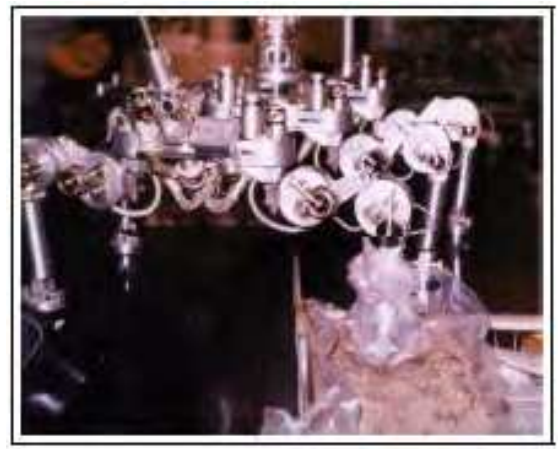

Fig. 2.1. A view of experiment "load-sinkage". 
The "load-sinkage" curves for different soils and some artificial materials were obtained experimentally In the experiments, all legs but one stayed on the rigid support; the remaining leg was placed on the soil for analysis (Fig.2.1). The load on this leg was repeatedly changed from zero to a maximum value (about $100 \mathrm{~N}$ ) and vice versa. Joint angle sensors determined leg sinkage and force sensors measured the load. The maximum load on the leg was 120 with a foot area of $30 \mathrm{~cm}^{2}$, equal to a specific pressure of $40 \mathrm{kPa}$. Some of the experimental "load-sinkage" relations of different soil types are shown in Fig.2.2a,b. As Fig. 2.2b and the literature make clear, sinkage in natural soils is irreversible. "Load-sinkage" relations for artificial materials are virtually unique and linear.

\subsection{Basic approaches to locomotion over soft soil}

The easiest way for a robot to walk over soft soil is to fix its locomotion cycles. The inhomogeneity of the soil's mechanical properties and the unevenness of the surface may disturb vehicle motion considerably.

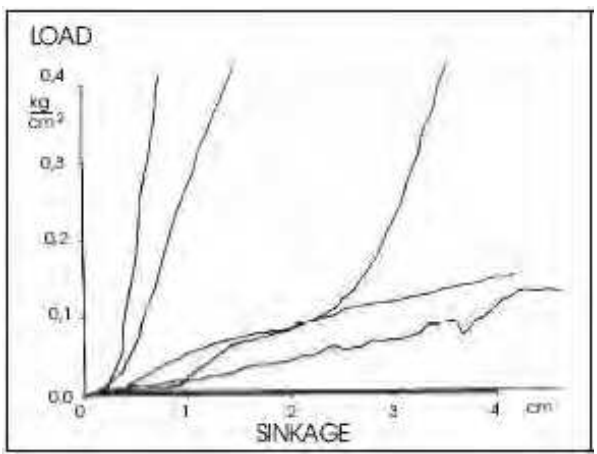

(a) "Load-sinkage" experiments: 1, 2 - rigid surfaces; 3 -sandwich-type sample; 4 flexible sample; 5 -foam-rubber

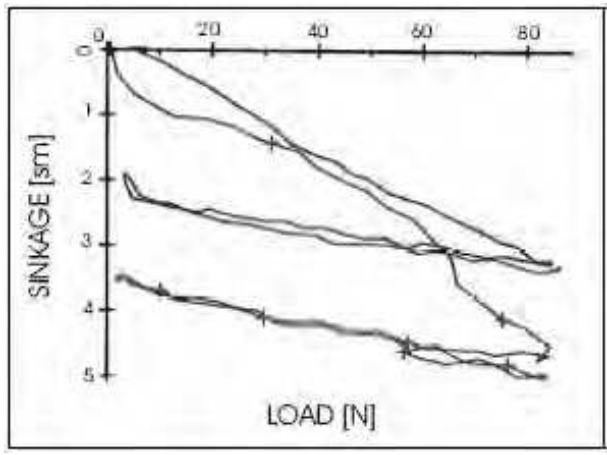

(b) "Load-sinkage" curves obtained by loading and unloading ground with a robot leg

Fig. 2.2. "Load-sinkage" curves.

A more complicated approach is a locomotion cycle with an adaptation zone (Gurfinkel et al., 1981). In order for a leg to strike the supporting surface in the adaptation zone, body position must be stabilized in relation to the support surface (roll, pitch and clearance), compensating for leg sinkage. To obtain smooth motion, the motion of each leg has to be corrected individually based on its sinkage.

Another demand on control algorithms is leg sinkage control in the joint support phase. If one of the support legs destroys the soil under it and sinks into the soil beyond the permissible level, the vehicle must be stopped and the sunken leg unloaded. A body's movements during vehicle motion over a non-rigid surface need to be stabilized in relation to the supporting surface. A variety of approaches can accomplish this.

The first approach is to control body movement along the normal to the surface. To do this, the position of the body in relation to the supporting surface (pitch, roll, clearance) must be known. In this case, the supporting legs are controlled in the same way as on a rigid surface, i.e. the supporting polygon remains stiff. 
However, most motion disturbance occurs within relatively short time periods when the set of supporting legs is changing. Therefore, to stabilize body movement, it is better to correct the programmed displacements of legs in relation to the body, corresponding to the setting of the legs into the soil. This is the second approach to stabilizing body movement during vehicle motion over soft soil.

\subsection{Motion control algorithms}

The sinkage of a leg into the soil depends on the load put on that leg. Accordingly, three methods to control leg sinkage suggest themselves.

The first method uses force feedback to control foot force reactions as shown in (Schneider, 1999). In this case, the stabilization of body movement requires allowing for a leg's sinkage into the soil when motion is generated. If the "load-sinkage" characteristics for each leg are known a priori, the sinkage can be computed from the programmed load on the leg. With this approach, the angular and the linear positions are corrected continuously during the motion.

The second method also assumes a priori knowledge of the "load-sinkage" characteristics for each leg. In this case, there is no force feedback and each leg's sinkage into the soil is controlled instead. To program sinkage, the corresponding force reactions must satisfy the static equilibrium equations.

The third and most complicated approach is to control bearing reactions and leg sinkage simultaneously. In this case, programmed leg motion is corrected for current leg sinkage into the soil. Although the mechanical properties of soil are not assumed to be known a priori in this case, the magnitude of sinkage into the soil must be known for each leg.

When soil is loaded, both reversible and irreversible deformations generally occur. To work out algorithms, let us assume that the relation of the sinkage to the applied load is the same for each point of the bearing area. Based on such an assumption, only the first two control methods, i.e. either control of bearing reactions or leg sinkage into the soil, have been worked out. Only two simplified types of soil shall be considered. For the first type of soil, all deformations are reversible and sinkage depends uniquely on load. Such an "elastic" surface might be found in a peat bog, for instance, where a layer of peat covers water. Although such situations are not widespread in nature, the problem of locomotion over elastic soil is of interest in and of itself.

The second type of soil has completely irreversible deformations. Most natural soils approximate this model. Such soil behaves as an absolutely rigid support if the load on the foot becomes less than a maximum value already achieved. The properties of naturally consolidating soils may differ considerably, even for adjacent points of terrain (Gorinevsky \& Shneider, 1990). Thus, the algorithm for motion control on such surfaces is based on the third method, which does not assume the soil characteristics are known a priori.

\subsubsection{Locomotion on linear elastic soil}

This algorithm is based on the assumption that the soil properties are known a priori. Let us assume that the force $N_{3}^{(i)}$ depends on the linear sinkage $s_{3}^{(i)}$ of the $i$-th leg as

$$
N_{3}^{(i)}=C_{s} s_{3}^{(i)},
$$

where soil stiffness $C_{s}$ is equal for all the legs and $N_{3}^{(i)}$ is the vertical foot force. 
Then, the motion of each supporting leg is corrected for its sinkage computed from the programmed foot force $N_{p}^{(i)}$

$$
S_{p}^{(i)}=N_{p}^{(i)} / C_{s}
$$

where the programmed foot-force ${ }^{N_{p}^{(i)}}$ is calculated using the force distribution algorithm of locomotion over a rigid surface.

\subsubsection{Locomotion on consolidating ground}

Let us assume that the mechanical properties of soil are not known a priori. Then the motion of each leg should be corrected for its sinkage. This cannot be computed beforehand. Rather, it can only be measured.

The algorithm is based on the assumption that soil deformation is completely irreversible. The leg may be considered to be on the rigid surface if the load on the leg is less than a maximum value already achieved. The absolute displacement of the body may then be determined from these leg positions.

Angular and linear displacements of the body must be known to determine leg sinkage into the soil. Let $\vec{R}^{(i)}\left(x_{1}^{(i)}, x_{2}^{(i)}, x_{3}^{(i)}\right)$ be the radius vector of the $i$-th leg in the body's fixed coordinate system, $\vec{R}\left(x_{0,1}^{(i)}, x_{0,2}^{(i)}, x_{0,3}^{(i)}\right)$ be the vector of displacements of the body's center in a world coordinate system and vector, $\vec{\varphi}(\psi, \theta)$ be small deviations of the roll and pitch of the body from its initial horizontal position and $\Delta H$ be a change in clearance resulting from leg sinkage.

Let $\vec{S}^{(i)}\left(s_{1}^{(i)}, s_{2}^{(i)}, s_{3}^{(i)}\right)$ denote the foot displacement of the $i$-th leg in the body's-fixed, and in the world coordinate systems, respectively. Then the following ensues

$$
\vec{S}^{(i)}=-\vec{\varphi} \times \vec{r}^{(i)}-\vec{R}+\vec{\sigma}^{(i)}
$$

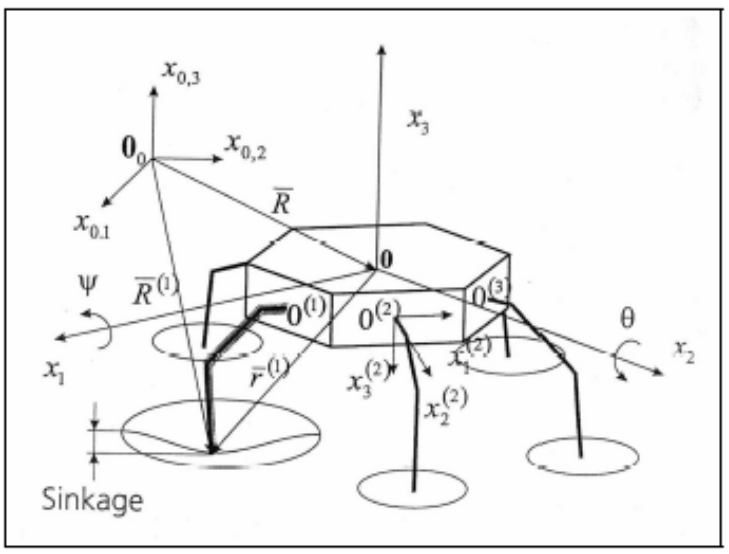

Fig. 2.3. Soil consolidation coordinate systems.

Let us assume that $n(n \geq 3)$ legs are on absolutely rigid soil. For these legs, $\sigma_{0,3}^{(i)}$ and equation (2.3) may be used to determine the angular displacements $(\psi, \theta)$ and linear displacements $\Delta H$ of the body. For a small vertical displacement $s_{3}^{(i)}$ of the leg after contact with soil, the foot displacement in the world 


$$
\sigma_{3}^{(i)}=\Delta H-\psi x^{(i)}+\theta y^{(i)}+s_{3}^{(i)}
$$

As the load on the legs is redistributed, the sinkage of the legs standing on soft soil will increase. Using information from the position sensors, the sinkage of each of these legs can be determined with equation (2.4). The angular $(\psi, \theta)$ and linear $(\Delta H)$ displacements of the body can be determined by applying the LaGrange multiplier method.

\subsection{Locomotion over consolidating ground with intermediate loading}

The sinkage control algorithm for moving on a non-rigid irreversibly deforming bearing surface (see section 6.3) has a number of disadvantages. In the transport phase, when the sinkage of supporting legs is not controlled, the displacement of the vehicle's center of mass may cause the load on the legs to increase. This may cause the legs to impact the soil uncontrollably and even the vehicle body to "land" on the soil or stability to be lost if some of the legs sink too deeply into the soil.

To circumvent this drawback, a walking algorithm with intermediate soil loading was developed.

During the joint support phase, each leg is loaded intermediately to make contact with soil up to a maximum achievable load. In this case, leg sinkage may exceed the permissible value only in the joint support phase when the body position is controlled by the remaining legs standing on the consolidated soil. After such loading, the soil under these legs may be considered fully consolidated.

For the algorithm to function successfully, provision must be made for redistributing the vertical support reaction components among the supporting legs in such a way that the maximum load would be on a leg planted on the surface. Several solutions to the problems of vertical foot force redistribution are known, e.g. (Okhotsimsky \& Golubev,1984; Waldron, 1986; Kumar \& Waldron, 1990) but the problem of load maximization for a given leg has obviously not been taken into account.

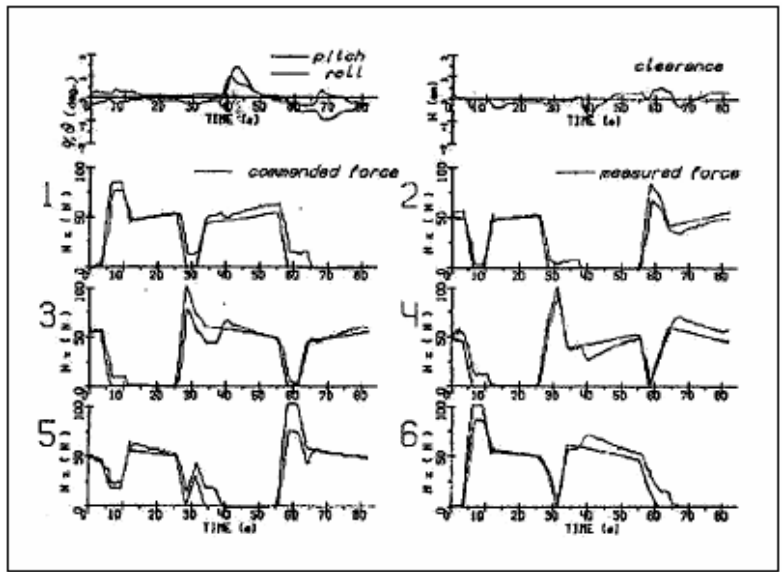

Fig. 2.4. Force distribution during locomotion on consolidating soft soil with intermediate loading (experiment with six-legged robot "Masha”).

The distribution of vertical reactions by three supporting legs is unique and can be calculated, for instance, with (Gorinevsky \& Shneider, 1990) if the number of supporting 
legs is given. A newly planted leg is loaded as follows. The supporting triples corresponding to a maximum loading of each newly planted leg are determined at the beginning of the full support phase. The support phase is divided into equal time intervals, where is the number of newly planted legs. During the first intervals, the forces are smoothly redistributed to load each of the newly planted legs. In the last interval, the legs to be transferred are unloaded.

The algorithm for walking robot motion over a soft surface with soil consolidation has been tested (Fig. 2.4). The vehicle moved with a diagonal gallop gait. Since two legs are placed on the soil at a time, the full support phase is divided into two parts: consolidation of soil under each of the two legs and transition to the final state (i.e. unloading the legs to be transferred). It can be seen that the qualitiy of tracking of the commanded forces and the stabilization of the body's motion has been improved.

\section{Force control of body motion for service operations}

\subsection{Definition of the force-moment vector by means of support reactions}

Synthesis of a control requires calculating the main vector of the force-moment arising from the process equipment's contact with an external object. The force-moment vector may be defined in two ways. A force sensor is placed between the robot body and the process equipment or the information from the force sensors in the robot legs is used. We shall consider the first method of calculating a force-moment vector.

Let the external force $\bar{F}$ at the point $O$ (Fig.3.1) be applied to the body of the legged robot standing on a rigid surface. The following orthogonal coordinate systems are introduced: the coordinate systems fixed to the surface $\left(O_{0} X_{01} X_{02} X_{03}\right)$, fixed to the body $\left(O X_{1} X_{2} X_{3}\right)$ and fixed to the attachment point of the legs $\left(O^{(i)} x_{1}^{(i)} x_{2}^{(i)} x_{3}^{(i)}\right)$.

Assuming the displacement of the body due to flexible deformation of the force sensors is insignificant, the influence of dynamic factors can be disregarded. Therefore, the quasi-static equations are applied to calculate $\bar{F}$ and $\bar{M}$. In this case, the condition for the robot's equilibrium is that all actual external forces and torques equal zero:

$$
\begin{gathered}
\sum_{i=1}^{n} \bar{N}^{(i)}+\bar{P}+\bar{F}=0, \\
\sum_{i=1}^{n}\left[\bar{r}^{(i)} \times \bar{N}^{(i)}\right]+\left[\bar{r}^{(i)} \times \bar{P}\right]+\bar{M}_{F}=0,
\end{gathered}
$$

where $\bar{N}^{(i)}$ is the vector of support reactions measured with the aid of force sensors mounted in the legs, $\bar{P}$ is the vehicle weight, $\bar{F}$ is the external force vector acting on the body (or on the tool mounted on the body) and $\bar{M}$ is the moment of external forces.

For the majority of operations performable by a manipulation robot or an adaptive legged platform, the geometric parameters of body and tools and the coordinate of the point of force application are assumed to be known. If the point of force application is known beforehand, the active external components of force and torque can be defined.

The equation (3.1) can be used to define three components $F_{x}, F_{y}, F_{z}$ of force vector $\bar{F}$. Accordingly, the directional cosines of vector $\bar{F}$ are

$$
\alpha=\frac{F_{x}}{|\bar{F}|}, \beta=\frac{F_{y}}{|\bar{F}|}, \gamma=\frac{F_{x}}{|\bar{F}|} \text {, where }|\bar{F}|^{2}=F_{x}^{2}+F_{y}^{2}+F_{z}^{2} .
$$




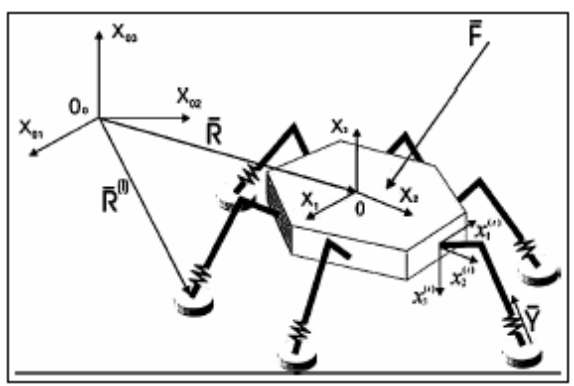

Fig. 3.1. Kinematics structure of the robot "Katharina".

If the external torque $\bar{M}$ is equal to zero, then the three scalar equations obtained with torque equation (3.2) can be used to define the coordinates of a point $A_{F}\left(a_{F X}, a_{F Y}, a_{F Z}\right)$ of applied external force $\bar{F}$.

To determine the main force $(\bar{F})$ and the torque $(\bar{M})$ in the coordinate system connected with the center of the body, the force reaction from axes $P^{(i)} y_{1}^{(i)} y_{2}^{(i)} y_{3}(i)$ and the linear displacement must be transformed to axes $O X_{1} X_{2} X_{3}$.

\subsection{Assembly operation by body displacement: Tube-in-hole insertion}

An assembly task is demonstrated here by inserting a tube with a diameter $d_{0}$ into a funnelshaped hole of an external object, the position of which is unknown (Fig.3.2).

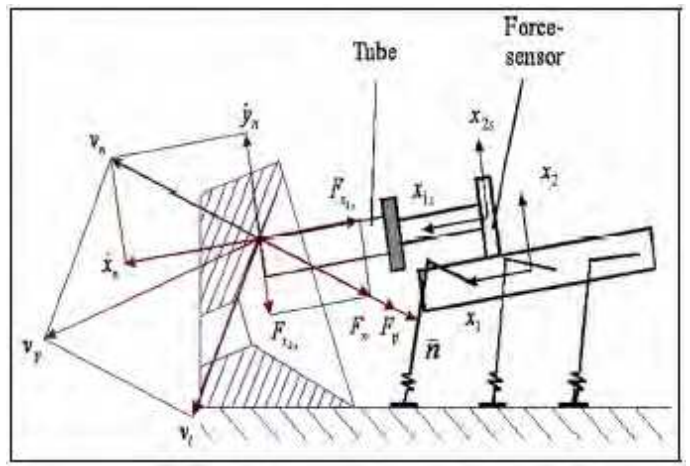

Fig. 3.2. Inserting a tube into a hole.

A force sensor rigidly connects the tube to the body of a hexapod vehicle. Its axial direction is parallel to the $O X_{1}$ axis, which is rigidly related to the body and its origin in the body's center. The external object's surface is a funnel-shaped hole with a diameter $d>d_{0}$. The task was studied using the linear motion of the robot's body and a method based on measuring the reaction force components generated by contact between the tube and the funnel-shaped surface.

The body of the robot is moved in such a way that the reaction forces are minimized. The tube moves toward the hole and touches the inside of the funnel. The force components are measured during this motion.

In this approach, force vector components are measured by establishing contact between the tube end and the funnel-shaped surface, maintaining a constant contact force equal to the programmed value and moving the robot's body with the tube in the direction of the hole. 
Two modes of force control have been investigated: independent translational body motions and the superposition of body motions. The elements of the accommodation matrix are adjusted so that they are large for movement's perpendicular to the hole and small for movements along the tube axis.

\subsubsection{An algorithm with accommodation of independent motions}

Components of the body's velocity vector $\bar{V}=\operatorname{col}\left(V_{X P}, V_{Y P}, V_{Z P}\right)$ were calculated conforming to the expression $\bar{V}_{p}=G_{F}\left(\bar{F}_{p}-\bar{F}\right)$, where $G_{F}=\left\|g_{i j}\right\|, \bar{F}$ is the measured value of contact force between the tube end and funnel-shaped surface and $\bar{F}_{p}$ is the commanded force. This algorithm transforms the motion of the body into three independent translational motions.

The experiments have demonstrated that a loss of contact can occur between the tube and the surface of the funnel-shaped hole. In the force reaction measurements, this phenomenon is observed as sudden changes of the force components, in particular changes of the longitudinal force component.

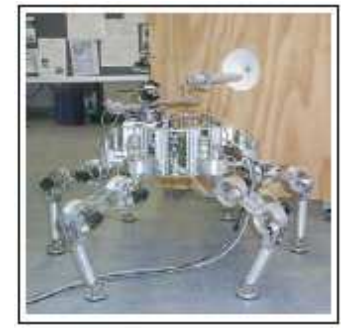

(a) Start position of robot

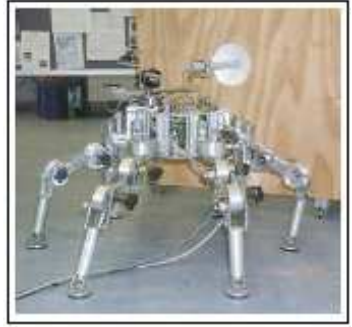

(b) End position of robot after insertion

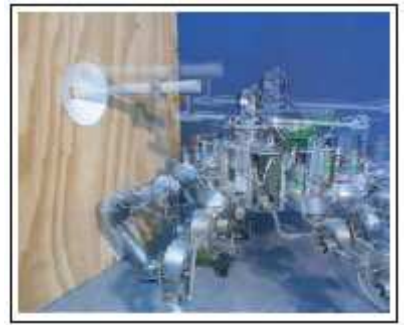

(c) Superposition of initial and finite positions

Fig. 3.3. Positions of the robot during the experiment.

\subsubsection{An algorithm with accommodation and superposition of motions}

This approach employed the control law as a superposition of two basic motions, i.e. motion normal and tangential to the funnel-shaped surface. A constraint was also added to the effect that the motion along a normal should maintain a constant force contact between the tube and the funnel surface. Accordingly, the programmed value of the tube velocity may be represented as the sum of the two components according to equation (1.18).

To utilize the control system described in section 4 , the value of the force $F_{n}$ as well as $\bar{n}$ and $\bar{\tau}$ which describe the direction of the body with the tube displacements must be evaluated. To determine $F_{n}, \bar{n}$, and $\bar{\tau}$, let us assume the longitudinal axis of the tube and the hole are collinear and that the tube moves over the funnel shape without friction.

Under the assumptions made, values $F_{n}$ and $\bar{n}$ can be evaluated with the help of the force sensor as in (Lensky et al., 1986; Gorinevsky et al., 1997).

Coefficients $V_{\tau}, F_{p}, \lambda$, and $F_{P X}$ have been selected experimentally: $V_{\tau}=0,7 \mathrm{~cm} / \mathrm{s}, F_{p}=30 \mathrm{~N}$, $\lambda=0,14 \mathrm{~cm} / \mathrm{sN}, F_{P X}=20 \mathrm{~N}$.

The results revealed that, in the case of the second control law, tube movement along the funnelshaped hole was more even and smooth. Contact was not lost between the tube and the funnelshaped surface. Fig.3.3a, $b$ and $c$ show positions of the robot during the experiment.

Obtained during the motion of the tube along the funnel-shaped hole force components $F-X, F_{y}, F_{z}$ are plotted in Fig.3.4. There, $x, y, z$ are displacements of the body and tube 
along axes $O X, O Y, O Z$ over time. Values of $\Delta x, \Delta y, \Delta z$ were calculated relative to the stationary feet standing on the surface. Other important values are the diameter of the tube $d=32 \mathrm{~mm}$, the diameter of the hole $d_{0}=40 \mathrm{~mm}$, the diameter of the funnel $\mathrm{D}=140 \mathrm{~mm}$, and the height of the funnel $h=50 \mathrm{~mm}$.

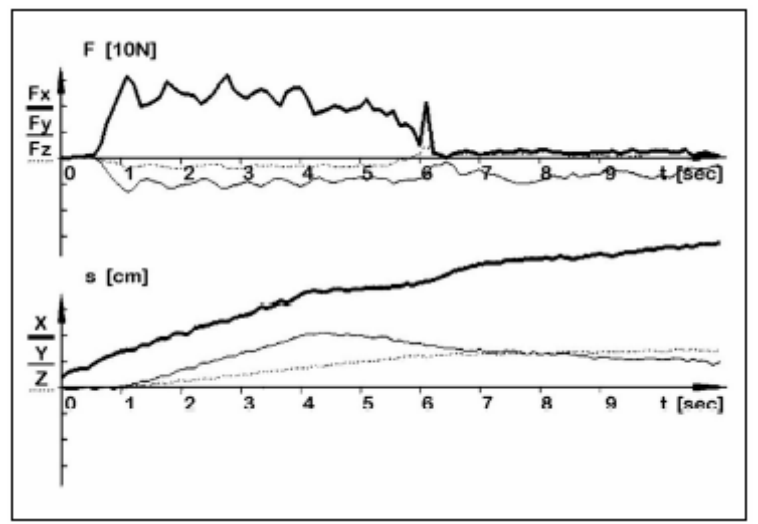

Fig. 3.4. Inserting a tube into a hole.

Some characteristic stages can be distinguished in the graph: The motion of the tube as it comes into contact with the funnel-shaped surface, the motion of the tube along this surface toward the hole and finally the insertion of the tube into the hole. After contact occurred between the tube's end and the surface, a force resulted and the program controlled the body's motion Contact was established between the tube and. the funnelshape with a normal force $F_{n}$ and the tube was moved along the conical surface. As the tube began to move into the hole, the value of the longitudinal force component $F_{x}$ decreased to zero. This was the signal to switch to accommodation control based on (1.18).

\subsection{Implementation of body motion control for a rotating a handle}

Let us consider a task of rotating a handle with a rod mounted with a force sensor on the body. Tasks similar to rotating a handle or steering a wheel by means of a manipulator were studied, e.g. in (De Schutter, 1986; Gorinevsky et al., 1997).

In our experiments, the force sensor is attached to the end of the manipulator arm fixture on the body of the legged robot. The other end of the sensor is connected to the tube that comes in contact with the handle. In this task, only two body translation degrees of freedom are used to control the motion of the handle and the manipulator is used as a bearing structure to which the tube is attached.

The position of the tube tip inserted in the grip of the handle is restricted to the circumference. Rotating the handle requires moving the body (together with the tube) along the circumference. In doing so, each point of the mast should move along a curve closely approximating a circle. We assume that the position of the circle's center and radius are not known a priori.

Therefore, to avoid a loss of contact between the tube and grip of the handle, the commanded force $F_{p}$ of contact with the object (see 1.18) must be positive. For the problem considered here, the constraint is binding (bilateral). Hence, using the same control expression (4.18) to solve this problem, we can take $F_{P}=0$ : 


$$
\bar{V}_{p}=g F_{n} \bar{n}+v \bar{\tau}
$$

In the preceding equation, $V_{p}=\left(V_{X P}, V_{Y P}\right.$ is a commanded velocity vector for the body together with the tube motion, $\bar{n}$ and $\bar{\tau}$ are vectors of normal and tangent to the constraint (circle), $F_{n}$ is the projection of the force applied to the sensor onto the normal $\bar{n}, v=$ const is the commanded velocity of the handle rotation (contouring velocity) and $g>0$ is a constant gain. The direction of rotation is determined by the direction of the vector $\bar{\tau}$ and sign of $v$. The normal $\bar{n}$ in (3.3) can be chosen as a vector directed either away from or toward the center of the handle.

If the friction in the handle axis and handle mass are negligible, then the force the handle exerts on the sensor is always in a direction tangent to the radius. Measuring the vector $\bar{F}$ of this force determines the vector of normal $\bar{n}=\bar{F} / F$ and thus the vector of tangent $\bar{\tau}$. Then we can rewrite (3.3) as follows:

$$
\bar{V}_{p}=g F+v \bar{\tau}
$$

When the force of resistance to handle rotation is nonzero but not too large, (3.4) may also be used.

If the load resistance of handle rotation is large, then (3.3) should be used to calculate the accommodated velocity vector. A priori information about friction in the handle axis may be used to calculate the vectors of normal and tangent to the constraint.

The position sensors in the legs can also be used to determine these vectors. As the handle rotates, each point of the tube moves together with the body along a curve closely approximating a circle. By measuring consecutive positions of the tube, a secant to the curve can be determined to build vectors close to $\bar{\tau}$ and $\bar{n}$. The vectors of normal and tangent should be given at the start of the motion.

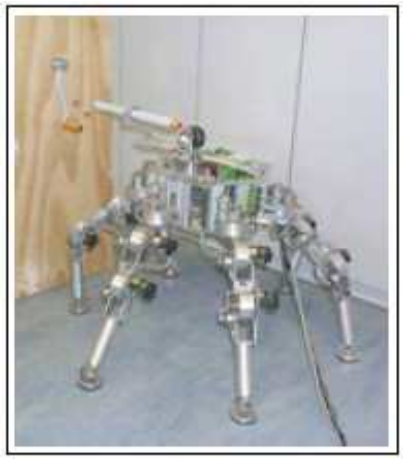

(a) Initial position

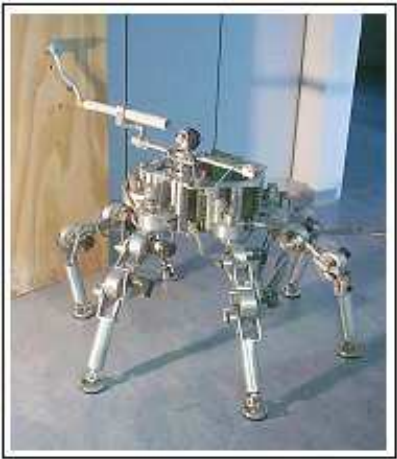

(b) Rotating of handle

Fig. 3.5. The experimental sheme.

The control laws presented were successfully applied in the experiments. The experimental scheme is shown in Fig. 3.5a (start position) and Fig. 3.5 b (rotation of handle).

\subsection{Drilling operation}

Another task was body motion for drilling operations. Using the body together with a drill as a working device requires controlling body movement in such a way that the longitudinal axis of the drill is collinear to the normal direction of a part's surface. Fig. 3.6a and 3.6b illustrate the initial and end positions of robot body orientation when drilling. 
At first, the robot moves parallel to the $O X$ axis to come in contact with the surface. The moment of contact is taken from the force sensor data. The body's displacement $X_{1}$ and clearance $H_{1}$ are evaluated at this moment. The change in the coordinates of the supporting leg ends describe the displacement.

Next, the body changes its clearance to $H_{2}$ through plane-parallel movements. Later, the body again moves in the axis $O X$. The body's displacement $X_{2}$ is evaluated at the moment of contact. The angle of the slope $\varphi$ of the working surface in relation to the $O_{1} X_{1} Z_{1}$ plane (angle of relation around $O_{1} Y_{1}$ ) is provided by:

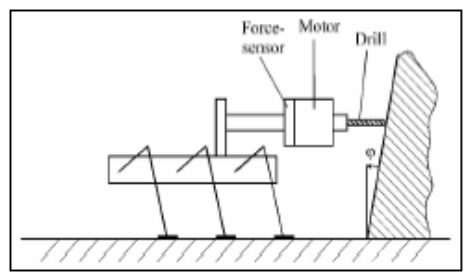

(a) Initial position of robot drilling

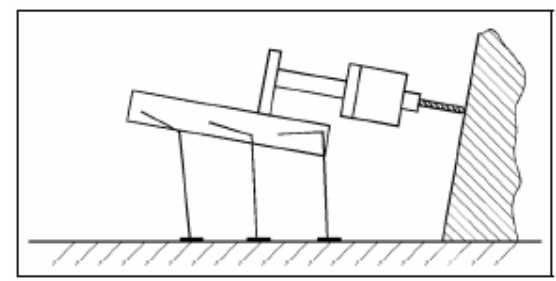

(b) End position of robot drilling

Fig. 3.6. Illustration of initial and end positions of robot body.

$$
\beta=\arctan \frac{X_{1}-X_{2}}{H_{1}-H_{2}},
$$

Next, the body changes its clearance to $H_{1}$ and moves parallel to the $O X$ axis to come into contact with the working surface. The programmed velocities of body rotation around the $O_{1} Y_{1}$ axis are

$$
\omega_{P Y}=k_{Y} \cdot \sin \beta, V_{P X}=\omega_{P Y} \cdot R,
$$

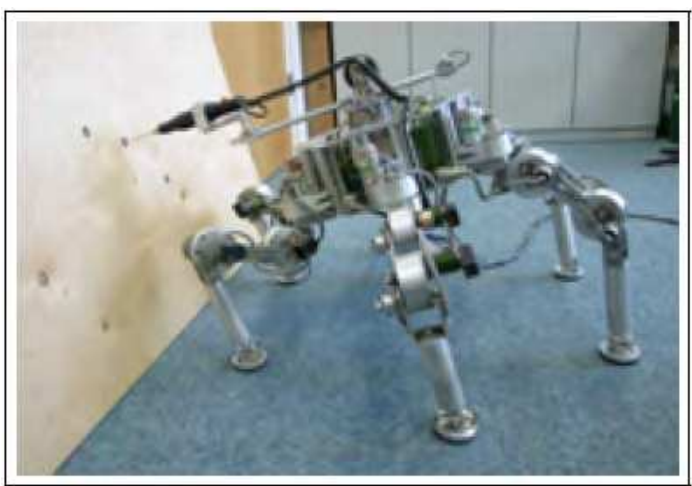

Fig. 3.7. Robot “Katharina" by drilling. 
The inclination sensor measures the actual tangential deviation $\theta$ of the body during its movement. When $(\varphi-\theta) \leq \Delta$ is smaller than the given value $\Delta$, the movement of the body is stopped. The tool then moves to touch the working surface. The direction of motion is parallel to the axis. The program controls the value of the force during contact. The program starts the drilling operation after initial contact.

In drilling operations, the pressing force determines the cutting force, which has to be controlled. The cutting force has to be constant in the direction of normal force $F_{N}$ at all times. This force has to be close to $F_{P X}$. To prevent jams and tool breakage, the control system can compensate for the lateral components of interaction forces that arise during the drilling operation. Force control of the body for technological operations has been developed and experimentally tested. Control of a moving body can be solved in terms of the force and torque vectors acting on the drill. If it is assumed that the commanded vectors $\bar{V}_{P}$ and $\bar{\omega}_{P}$ of the body depend on the force and torque expressed as:

$$
V_{P X}=k_{X}\left(F_{X}-F_{P X} ; \omega_{P Y}=-k_{Z} F_{Z} ; \omega_{P Z}=k_{Y} F_{Y} ; V_{P Y}=-\omega_{P Z} R ; V_{P Y}=-\omega_{P Z} R,\right.
$$

where $F_{X}, F_{Y}, F_{Z}$ force components are measured by the force sensor, $k_{X}, k_{Y}, k_{Z}$ is the feedback gain and $R$ is a constant describing the distance between the coordinate frame and drill end, then the vehicle body will move in a specified direction.

The accuracy of the preceding control algorithm cannot be expected to be very high because of the contact friction between the drill end and the working surface. To improve this situation a more effective algorithm was elaborated based on:

- Measuring several position points on the working surface,

- Evaluating the slope angle of this surface in relation to the fixed coordinate frame and the angles (pitch and course) of the vehicle body at the first point of contact between the drill and the working surface. The longitudinal displacement of a drill and the reaction to force components during drilling experiments are plotted in Fig. 3.8.

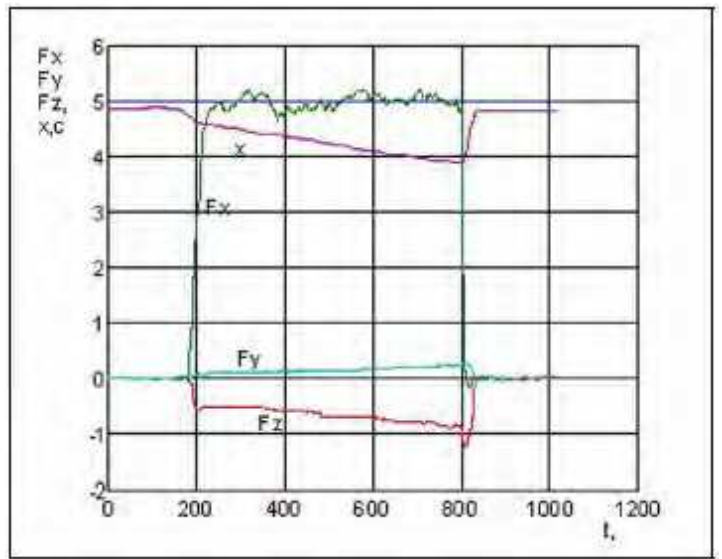

Fig. 3.8. Plots of the longitudinal displacement $(x)$ and force components $\left(F_{x}, F_{y}, F_{z}\right)$.

However, the experiments also demonstrated that sustained oscillations arise in the mechanical system whenever force feedback gains are large or force sensor stiffness is high and the feedback loop has a time delay. A simplified mathematical model of translation motion of the manipulator together with the drill maintaining contact with a rigid object 
(wooden plate) is described in (Schneider et al., 2004). This model was used to derive the stability criteria of the "'drill-manipulator"', system for the legged robot.

\section{Acknowledgements}

Initial research began at the Institute of Mechanics together with the Institute for Problems of Information Transmission (Moscow) at the initiative of Professors D.E. Okhotsimsky, V.S. Gurfinkel, members of the Russian Academy of Sciences and Professor E.A. Devjanin. Research is continuing at the Fraunhofer Institute for Factory Operation and Automation (IFF, Magdeburg) and is being supported by the Germany Research Foundation DFG.

We would like to express special thanks to Professor D. M. Gorinevsky, together with whom the basic results of the force distribution algorithms have been achieved and also Professors A. Formalsky, and Yu. Golubev. The author is deeply grateful to Doctors A. Lensky, E. Gurfinkel and L. Shtilman.

\section{Summary}

Years of research and experimental development of sensing system and control algorithms allow drawing the following conclusions:

- The devices and control algorithms developed can be used as a basis for developing both new laboratory models and full-scale walking robots, their sensors, force systems and control algorithms;

- The implementation of impedance force control (e.g. active compliance, active accommodation, hybrid position/force control) simplifies the series of algorithms and increases the fields of application for walking robots;

- The information on foot force interactions between robot legs and a surface used by the control system improves adaptation to terrain roughness and provides for uniform distribution of forces between supporting legs;

- The control systems based on interaction force information are suited for solving the problem of moving a legged robot or displacing a body along a constraint (most applications require this type of control).

The information on the main force and the torque vectors acting on the vehicle body coming into contact with an external object and maintaining the specified contact force is used for assembly and drilling operations.

\section{References}

Alexandre, P., Doroftei, I. \& Preumont, A. (1998). An autonomous micro walking machine with articulated body. In 3rd IFAC Symp. on Intell. Autonom. Vehicles, pp. 61-66. Madrid.

Bekker, M. (1969). Introduction to Terrain-Vehicle Systems. University of Michigan Press,.

Cruse, H., Kindermann, T., Schumm, M., Dean, J. \& Schmitz, J.(1998). Walknet-a biologically inspired network to control six-legged walking. Neural Networks 11, 1435-1447.

De Schutter, J.(1986). Compliant robot motion: Task formulation and control. PhD thesis, Katholieke Univ. Leuven.

De Schutter, J. \& Brussel, H. V.(1988). Compliant robot motion. Inter. J. Robot. Res. 7 (4), 333. 
Devjanin, E., Gurfinkel, V., Gerfinkel, E., Kartashev, V., Lensky, A., Shneider, A. \& Shtilman, L. (1983). The six-legged walking robot capable of terrain adaptation. Mechanism and Machine 18 (4), 257-260.

Devjanin, E., Kartashev, V., Lensky, A. \& Schneider, A. Y.(1982). "Investigation of robotics", chap. Force feedback control of legged vehicle, pp. 147-158. Moscow (in Russian): Press "Nauka".

Frik, M. (1996). Adaptive neural control of a walking machine. In 7th German-Japanese Seminar on Nonlinear Problems in Dynamical Systems -Theory and Applications, pp. 141-148. Reisenburg, Germany.

Gantmacher, F.(1960). The Theory of Matrices. N.Y.: Chelsea Publishing Company.

Gaurin, G. (1994). Control of walking robots on natural terrain. PhD thesis, ETH, Zurich, No.10898.

Golubev, Y., Kartashov, V. \& Schneider, A.(1979). 2 All-Union conference "Beomechanics Problems", chap. Force distribution in legs of legged robot, pp. 152-153. Riga, (in Russian): "Zinatne" Press.

Gorinevsky, D., Formalsky, A. \& Schneider, A. (1997). Force Control of Robotics Systems, p. 350. N.Y.: CRC Press.

Gorinevsky, D. \& Shneider, A. (1987). Dynamics of small motion of a walking robot when there is feedback with respect to the support reactions. Mechanics of Solids 22 (6), 3746.

Gorinevsky, D. \& Shneider, A. (1990). Force control in locomotion of legged vehicle over rigid and soft surfaces. Int. J. Robot. Res. 9 (2), 4-23.

Gurfinkel, V., Devjanin, E., Lensky, A., Mozhevelov, S., Formalsky, A. \& Schneider, A. (1984). Force feedback in manipulator control system. Mechanics of Solids 19 (6), 52 59.

Gurfinkel, V., Gurfinkel, E., Devjanin, E., Efremov, E., Zhicharev, D., Lensky, A., Schneider, A. \& Shtilman, L. (1982). "Investigation of Robotics", chap. Six-legged walking model of vehicle with supervisory control, pp. 98-147. Moscow, (in Russian).: Press "Nauka".

Gurfinkel, V., Gurfinkel, E., Schneider, A., Devjanin, E., Lensky, A. \& Shtilman, L. (1981). Walking robot with supervisory control. Mechanism and Machine Theory $16,31-36$.

Haykin, S. (1994). Neural Networks. Prentice Hall.

Hogan, N. (1985). Impedance theory control - an approach to manipulation. Trans. ASME J. of Dynam. Syst.,Measur. and Control, 107 (1), 1-24.

Kazerooni, H., Hout, P. \& Sheridan, T.(1986). Robust compliant motion for manipulators, part 1 and part 2. Trans. of IEEE J. Robotics and Autom. 2 (2), 83-105.

Kemurdjian, A. \& et.al. (1993). Planet Rovers, p. 319. Moscow, (in Russian): Mashinostroenie.

Klein, C. \& Briggs, R.(1980). Use of active compliance in the control of legged vehicles. IEEE Trans. Sys. Man Cybernet SMC-10 (7), 393-400.

Klein, C. \& Wahavisan, W.(1984). Use of a multiprocessor for control of a robotic system. Int. J. Robot. Res. 1 (2), 45-59.

Kumar, V. \& Waldron, K. (1990). Force distribution in walking vehicles. ASME J. of Mechanical Design 112 (Mach.), 90-99.

Lensky, A., Lizunov, A., Formalsky, A. \& Schneider, A. (1986). Manipulator motion along a constraint. Robotica 4 (4), 247-253. 
Lin, Y. \& Song, S.-M.(1997). Learning hybrid position/force control of a quadruped walking machine using a cmac neural network. J. Robotic Systems 14 (6), 483-499.

Manko, D.(1992). A General Model of Legged Locomotion on Natural Terrain, p. 113. Kluwer Academic Publishers.

Mason, M. \& Salisbury, J.(1985). Robot hands and the mechanics of manipulation.. Cambridge, Mass: MIT Press.

McGhee, R., Olson, K. \& Briggs, R. (1980). Proc. of 1980 Automotive Engineering Congress. Detroit, Michigan, chap. Electronic Coordination of Joint Motion for TerrainAdaptive Robot Vehicles, pp. 1-7. N.Y.: Pergamon Press.

Okhotsimsky, D. \& Golubev, Y.(1984). Motion mechanics and control of motion an automated walking vehicle, p. 312. Moscow, (in Russian): Publishing House "Nauka".

Palis, F., Rusin, V. \& Schneider, A. (2001). Adaptive impedance/force control of legged robot systems. In 4th Int. Conf. on Climbing and Walking Robots, CLAWAR'01, pp. 324-330. Karlsruhe.

Raibert, M. \& Craig, J. (1981). Hybrid position/force control of manipulators. ASME J. of Dynam. Syst., Measur. and Control 103 (2), 126-133.

Salisbury, J. \& Craig, J.(1982). Articulated hands: force control and kinematics issues. Int. J. of Robot. Res. 1 (1), 4-17.

Schmucker, U., Schneider, A. \& Ihme, T. (1997). Force control for legged robots. In ICAR'978th Int. Conf. on Advanced Robotics. Workshop "New Approaches on Dynamic Walking and Climbing Machines", pp. 38-43. Monterey, USA.

Schneider, A.(1999). Control algorithms of interaction between walking robot legs and soft soil. In 2nd Inter. Conf. on Climbing and Walking Robots, CLAWAR'99, pp. 473-481. Pothsmouth, UK.

Schneider, A. \& Schmucker, U. (2000). Adaptive six-legged platform for mounting and service operation. In 3rd Int. Conf. on Climbing and Walking Robots, CLAWAR'00, pp. 193-200. Madrid.

Schneider, A. \& Schmucker, U. (2001). Force legged platform "katharina" for service operations. In 4 Inter. Conf. on Climbing and Walking Robots, CLAWAR'01, pp. 10291036. Karlsruhe, Germany.

Schneider, A., Zeidis, I. \& Zimmermann, K. (2004). Stability of a "manipulator-drill" system with force control and time delay. Technische Mechanik 24 (1), 51-60.

Sciavicco, L. \& Siciliano, B. (2000). Modelling and Control of Robot Manipulators, p. 378. Springer Verlag.

Sinha, P. \& Goldenberg, A. (1993). A unified theory for hybrid control of manipulator. In IEEE Int. Conf. on Robotics and Automation, pp. 343-348.

Surdilovic, D. \& Vukobratovic, M.(2001). Mechanical Systems Design Handbook, chap. Control of robotic systems in contact tasks, pp. 587-638. N.Y.: CRC Press.

Tzafestas, C., Guihard, M. \& M'Sirdi, K. (1995). Two-stage adaptive impedance control applied to a legged robot. In Int. Conf. on Intelligent Robots and Systems, pp. 173-178.

Waldron, K. (1986). Force and motion management in legged locomotion. IEEE J. of Robot. Automat. 2 (4), 214-220.

Whitney, D. (1977). Force feedback control of manipulator fine motions. Trans. ASME J. of Dynam. Syst., Measur. and Control 99 (2), 91-97.

Wong, J. (1993). Theory of Ground Vehicles. N.Y.: John Wiley \& Sons, Inc. 


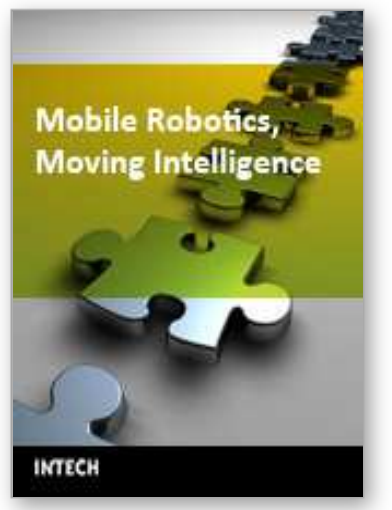

\author{
Mobile Robotics, Moving Intelligence \\ Edited by Jonas Buchli
}

ISBN 3-86611-284-X

Hard cover, 586 pages

Publisher Pro Literatur Verlag, Germany / ARS, Austria

Published online 01, December, 2006

Published in print edition December, 2006

This book covers many aspects of the exciting research in mobile robotics. It deals with different aspects of the control problem, especially also under uncertainty and faults. Mechanical design issues are discussed along with new sensor and actuator concepts. Games like soccer are a good example which comprise many of the aforementioned challenges in a single comprehensive and in the same time entertaining framework. Thus, the book comprises contributions dealing with aspects of the Robotcup competition. The reader will get a feel how the problems cover virtually all engineering disciplines ranging from theoretical research to very application specific work. In addition interesting problems for physics and mathematics arises out of such research. We hope this book will be an inspiring source of knowledge and ideas, stimulating further research in this exciting field. The promises and possible benefits of such efforts are manifold, they range from new transportation systems, intelligent cars to flexible assistants in factories and construction sites, over service robot which assist and support us in daily live, all the way to the possibility for efficient help for impaired and advances in prosthetics.

\title{
How to reference
}

In order to correctly reference this scholarly work, feel free to copy and paste the following:

A. Schneider and U. Schmucker (2006). Force Sensing for Multi-Legged Walking Robots: Theory and Experiments Part 2: Force Control of Legged Vehicles, Mobile Robotics, Moving Intelligence, Jonas Buchli (Ed.), ISBN: 3-86611-284-X, InTech, Available from:

http://www.intechopen.com/books/mobile_robotics_moving_intelligence/force_sensing_for_multilegged_walking_robots_theory_and_experiments_part_2_force_control_of_legg

\section{INTECH}

open science | open minds

\section{InTech Europe}

University Campus STeP Ri

Slavka Krautzeka 83/A

51000 Rijeka, Croatia

Phone: +385 (51) 770447

Fax: +385 (51) 686166

www.intechopen.com

\section{InTech China}

Unit 405, Office Block, Hotel Equatorial Shanghai

No.65, Yan An Road (West), Shanghai, 200040, China

中国上海市延安西路 65 号上海国际贵都大饭店办公楼 405 单元

Phone: +86-21-62489820

Fax: +86-21-62489821 
(C) 2006 The Author(s). Licensee IntechOpen. This chapter is distributed under the terms of the Creative Commons Attribution-NonCommercialShareAlike-3.0 License, which permits use, distribution and reproduction for non-commercial purposes, provided the original is properly cited and derivative works building on this content are distributed under the same license. 